\title{
Precise measurement of the positive muon anomalous magnetic moment
}

\author{
H.N. Brown ${ }^{2}$, G. Bunce ${ }^{2}$, R.M. Carey ${ }^{1}$, P. Cushman ${ }^{9}$, G.T. Danby ${ }^{2}$, P.T. Debevec ${ }^{7}$, M. Deile ${ }^{11}$, H. Deng ${ }^{11}$, \\ W. Deninger ${ }^{7}$, S.K. Dhawan ${ }^{11}$, V.P. Druzhinin ${ }^{3}$, L. Duong ${ }^{9}$, E. Efstathiadis ${ }^{1}$, F.J.M. Farley ${ }^{11}$, G.V. Fedotovich ${ }^{3}$, \\ S. Giron ${ }^{9}$, F. Gray ${ }^{7}$, D. Grigoriev ${ }^{3}$, M. Grosse-Perdekamp ${ }^{11}$, A. Grossmann ${ }^{6}$, M.F. Hare ${ }^{1}$, D.W. Hertzog ${ }^{7}$, \\ V.W. Hughes ${ }^{11}$, M. Iwasaki ${ }^{10}$, K. Jungmann ${ }^{6}$, D. Kawall ${ }^{11}$, M. Kawamura ${ }^{10}$, B.I. Khazin ${ }^{3}$, J. Kindem ${ }^{9}$, \\ F. Krienen ${ }^{1}$, I. Kronkvist ${ }^{9}$, R. Larsen ${ }^{2}$, Y.Y. Lee ${ }^{2}$, I. Logashenko ${ }^{1,3}$, R. McNabb ${ }^{9}$, W. Meng ${ }^{2}$, J. Mi ${ }^{2}$, J.P. Miller ${ }^{1}$, \\ W.M. Morse ${ }^{2}$, D. Nikas ${ }^{2}$, C.J.G. Onderwater ${ }^{7}$, Y. Orlov ${ }^{4}$, C.S. Özben ${ }^{2}$, J.M. Paley ${ }^{1}$, C. Polly ${ }^{7}$, J. Pretz ${ }^{11}$, R. Prigl ${ }^{2}$, \\ G. zu Putlitz ${ }^{6}$, S.I. Redin ${ }^{11}$, O. Rind ${ }^{1}$, B.L. Roberts ${ }^{1}$, N. Ryskulov ${ }^{3}$, S. Sedykh ${ }^{7}$, Y.K. Semertzidis ${ }^{2}$, \\ Yu.M. Shatunov ${ }^{3}$, E.P. Sichtermann ${ }^{11}$, E. Solodov ${ }^{3}$, M. Sossong ${ }^{7}$, A. Steinmetz ${ }^{11}$, L.R. Sulak ${ }^{1}$, C. Timmermans ${ }^{9}$, \\ A. Trofimov ${ }^{1}$, D. Urner ${ }^{7}$, P. von Walter ${ }^{6}$, D. Warburton ${ }^{2}$, D. Winn ${ }^{5}$, A. Yamamoto ${ }^{8}$, D. Zimmerman ${ }^{9}$ \\ Muon $(g-2)$ Collaboration \\ ${ }^{1}$ Department of Physics, Boston University, Boston, MA 02215, USA ${ }^{2}$ Brookhaven National Laboratory, Upton, NY 11973, \\ USA ${ }^{3}$ Budker Institute of Nuclear Physics, Novosibirsk, Russia ${ }^{4}$ Newman Laboratory, Cornell University, Ithaca, NY 14853, \\ USA ${ }^{5}$ Fairfield University, Fairfield, CT 06430, USA ${ }^{6}$ Physikalisches Institut der Universität Heidelberg, 69120 Heidelberg, \\ Germany ${ }^{7}$ Department of Physics, University of Illinois at Urbana-Champaign, IL 61801, USA ${ }^{8}$ KEK, High Energy \\ Accelerator Research Organization, Tsukuba, Ibaraki 305-0801, Japan ${ }^{9}$ Department of Physics, University of Minnesota, \\ Minneapolis, MN 55455, USA ${ }^{10}$ Tokyo Institute of Technology, Tokyo, Japan ${ }^{11}$ Department of Physics, Yale University, \\ New Haven, CT 06520, USA
}

(February 23, 2001)

\begin{abstract}
A precise measurement of the anomalous $g$ value, $a_{\mu}=(g-2) / 2$, for the positive muon has been made at the Brookhaven Alternating Gradient Synchrotron. The result $a_{\mu^{+}}=11659$ 202(14)(6) $\times$ $10^{-10}(1.3 \mathrm{ppm})$ is in good agreement with previous measurements and has an error one third that of the combined previous data. The current theoretical value from the standard model is $a_{\mu}(\mathrm{SM})=11659159.6(6.7) \times 10^{-10}(0.57 \mathrm{ppm})$ and $a_{\mu}(\exp )-a_{\mu}(\mathrm{SM})=43(16) \times 10^{-10}$ in which $a_{\mu}(\exp )$ is the world average experimental value.

PACS number: 14.60.Ef 13.40.Em
\end{abstract}

Precise measurement of the anomalous $g$ value, $a_{\mu}=(g-2) / 2$, of the muon provides a sensitive test of the standard model of particle physics and new information on speculative theories beyond it. Compared to the electron, the muon $g$ value is more sensitive to standard model extensions, typically by a factor of $\left(m_{\mu} / m_{e}\right)^{2}$. In this Letter we report a measurement of $a_{\mu}$ for the positive muon from Brookhaven AGS experiment 821, based on data collected in 1999.

The principle of the experiment, previous results, and many experimental details have been given in earlier publications [1].2]. Briefly, highly polarized $\mu^{+}$of $3.09 \mathrm{GeV} / c$ from a secondary beamline are injected through a superconducting inflector [3] into a storage ring $14.2 \mathrm{~m}$ in diameter with an effective circular aperture $9 \mathrm{~cm}$ in diameter. The superferric storage ring [1] has a homogeneous magnetic field of $1.45 \mathrm{~T}$, which is measured by an NMR system relative to the free proton NMR frequency [5.6.6. Electrostatic quadrupoles provide vertical focusing. A pulsed magnetic kicker gives a 10 mrad deflection which places the muons onto stored orbits. The muons start in $50 \mathrm{~ns}$ bunches and debunch with a decay time of about $20 \mu$ s due to their $0.6 \%$ momentum spread. Positrons are detected using 24 lead/scintillating fiber electromagnetic calorimeters [7] read out by waveform digitizers. The waveform digitizer and NMR clocks were phase-locked to the Loran $\mathrm{C}$ frequency signal.

The muon spin precesses faster than its momentum rotates by an angular frequency $\omega_{a}$ in the magnetic field $\langle B\rangle$ weighted over the muon distribution in space and time. The quantity $a_{\mu}$ is

$$
a_{\mu}=\frac{\omega_{a}}{\frac{e}{m_{\mu} c}\langle B\rangle},
$$

where $\omega_{a}$ is unaffected by the electrostatic field for muons with $\gamma=29.3$. Parity violation in the decay $\mu^{+} \rightarrow$ $e^{+} \overline{\nu_{\mu}} \nu_{e}$ causes positrons to be emitted with an angular and energy asymmetry. Because of the Lorentz boost, the positron emission angle with respect to the muon spin direction in the muon rest frame is strongly coupled to its energy in the laboratory frame. The number of decay positrons with energy greater than $E$ is described by

$$
N(t)=N_{0}(E) e^{-t /(\gamma \tau)}\left[1+A(E) \sin \left(\omega_{a} t+\phi_{a}(E)\right)\right]
$$

in which the time dilated lifetime $\gamma \tau \approx 64.4 \mu \mathrm{s}$. Some $140 \mathrm{~g}-2$ periods of $4.37 \mu$ s were observed.

Most experimental aspects of the data taking in 1999 were the same as in 1998 [1]. However, some improvements were made. Care was taken in tuning the AGS ejection system to minimize background from any extraneous proton beam extracted during the muon storage time. Scintillating fiber detectors which could be moved in and out of the storage region were used to study beam properties. Scintillation counters in front of five calorimeters were used to measure muon losses from the storage ring. 
The principal new feature of the 1999 run is the 20fold increase in data collected. During a data acquisition time of 500 hours, we obtained about 2.9 billion decay positrons with energies greater than $1.0 \mathrm{GeV}$. The AGS delivered $4 \times 10^{13}$ protons of $24 \mathrm{GeV}$ in six bunches, separated by $33 \mathrm{~ms}$, over its $2.5 \mathrm{~s}$ cycle. This resulted in $5 \times 10^{4}$ stored muons per cycle.

The magnetic field $B$ is obtained from NMR measurements of the free proton resonance frequency $\omega_{p}$. Seventeen NMR probes are mounted in an array on a trolley which moves on a fixed track inside the muon storage ring vacuum. The trolley probes are calibrated with respect to a standard spherical $\mathrm{H}_{2} \mathrm{O}$ probe to an accuracy of $0.2 \mathrm{ppm}$ before and after data-taking periods. Interpolation of the field in the periods between trolley measurements, which are made on average every three days, is based on the readings of about 150 fixed NMR probes distributed around the ring in the top and bottom walls of the vacuum chamber. Figure 1 shows a magnetic field profile averaged over azimuth. The variations in the amplitudes of the multipoles affect $\langle B\rangle$ by less than $0.02 \mathrm{ppm}$. The average readings of 36 uniformly distributed fixed probes are maintained to $0.1 \mathrm{ppm}$ by feedback to the main magnet power supply.

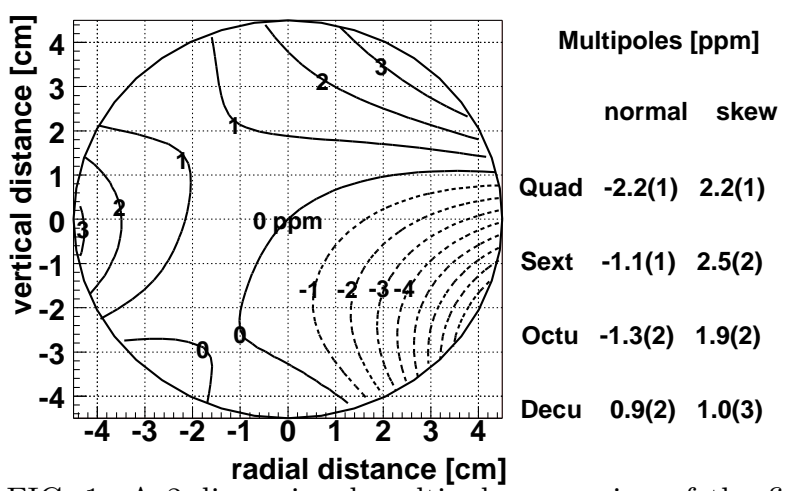

FIG. 1. A 2-dimensional multipole expansion of the field averaged over azimuth from one out of seventeen trolley measurements. One ppm contours are shown with respect to a central azimuthal average field $B_{0}=1.451266 \mathrm{~T}$. The circle indicates the muon beam storage region. The multipole amplitudes at the beam aperture radius of $4.5 \mathrm{~cm}$ are given.

The 1.45 $\mathrm{T}$ magnetic field of the superconducting inflector is well shielded from the storage region by flux trapping. However, over an azimuthal angle of $\sim 1^{\circ}$, a residual fringe field reduces the storage ring central field by about $600 \mathrm{ppm}$, increasing to $3000 \mathrm{ppm}$ at the edge of the aperture. This fringe field reduces the field homogeneity at large positive radial and negative vertical distances (Fig. 11).

The magnetic field is weighted with the muon distribution. Four thousand simulated muons were tracked for 100 turns through a field mapped by trolley measurements to evaluate the field muons encounter. The result agrees with $B$ measured with the trolley probes, averaged over the azimuth, and taken at the beam center.

Two largely independent analyses of $\widetilde{\omega}_{p}$, which is proportional to $\langle B\rangle$, were made using different selections of NMR probes. Their results agree to within $0.03 \mathrm{ppm}$. The final value is $\widetilde{\omega}_{p} / 2 \pi=61791256 \pm$ $25 \mathrm{~Hz}(0.4 \mathrm{ppm})$. Table I lists the systematic errors.

TABLE I. Systematic errors for the $\widetilde{\omega}_{p}$ analysis

\begin{tabular}{l|c}
\hline \hline Source of errors & Size [ppm] \\
\hline Absolute calibration of standard probe & 0.05 \\
Calibration of trolley probes & 0.20 \\
Trolley measurements of $B_{0}$ & 0.10 \\
Interpolation with fixed probes & 0.15 \\
Inflector fringe field & 0.20 \\
Uncertainty from muon distribution & 0.12 \\
Others $\dagger$ & 0.15 \\
\hline Total systematic error on $\widetilde{\omega}_{p}$ & 0.4 \\
\hline \hline
\end{tabular}

$\bar{\dagger}$ higher multipoles, trolley temperature and its power supply voltage response, and eddy currents from the kicker.

The frequency $\omega_{a}$ is obtained from the time distribution of decay $e^{+}$counts. The $e^{+}$are detected by calorimeters whose photomultiplier signals have a typical FWHM of $5 \mathrm{~ns}$. The signals are sampled every $2.5 \mathrm{~ns}$ by 8 -bit waveform digitizers (WFD) with at least 16 samples per $e^{+}$event. The samples are used to determine $e^{+}$times and energies, and for pulse overlap studies. The pulsereconstruction algorithm fits signals above baseline to an average pulse shape, determined for each calorimeter individually. Multiple pulses can be resolved if their separation exceeds 3 to 5 ns. Systematic effects associated with the algorithm were extensively studied.

Whereas for the 1998 data, muon decay and spin precession (Eq. 22) was adequate to describe the $e^{+}$time spectrum, the higher count rate and much larger data sample for 1999 required careful consideration of (1) $e^{+}$ pulses overlapping in time (pileup), (2) coherent betatron oscillations, (3) beam debunching, (4) muon losses, and (5) detector gain stability during the muon storage time.

(1) The number of pileup pulses in the reconstructed data is proportional to the instantaneous counting rate squared and to the minimum pulse separation time of the reconstruction algorithm. It amounts to $1 \%$ when the fits of $\omega_{a}$ to the data are started, and distorts the $e^{+}$time spectrum because of misidentification of the number, energies, and times of the positrons. Since the phase $\phi_{a}(E)$ (cf. Eq. 2) depends on the energies of the positrons, pileup has a phase which differs from $\phi_{a}$ leading to an error in $\omega_{a}$. Therefore, the data are corrected prior to fitting by subtracting a constructed pileup spectrum. Positrons found within a window at a fixed time after the pulse that triggers the WFD are treated as if they overlap with the trigger pulse. The width of the window is taken equal to the minimum pulse separation time. Only data with energies at least twice the hardware threshold 
are fully corrected with this method. Our $1 \mathrm{GeV}$ hardware threshold leads to a choice of $E \geq 2 \mathrm{GeV}$ in the $\omega_{a}$ analysis.

The contribution to pileup from signals too small to be reconstructed is not accounted for by the procedure described above. These small signals distort the pulse reconstruction but do not, on average, affect the energy. However, such unseen pulses introduce small timedependent shifts in $\phi_{a}(E)$ and $A(E)$. The time dependence of the asymmetry, being more sensitive than the phase, is used to set a limit on the shift of $\omega_{a}$.

Fig. 2 shows the agreement between the positron energy spectrum after pileup subtraction and the spectrum when pileup is negligible, together with the uncorrected spectrum. The inset illustrates that the average energy after pileup subtraction is constant with time, as expected. Without accounting for pileup, a shift in $\omega_{a}$ of $0.3 \mathrm{ppm}$ would result.

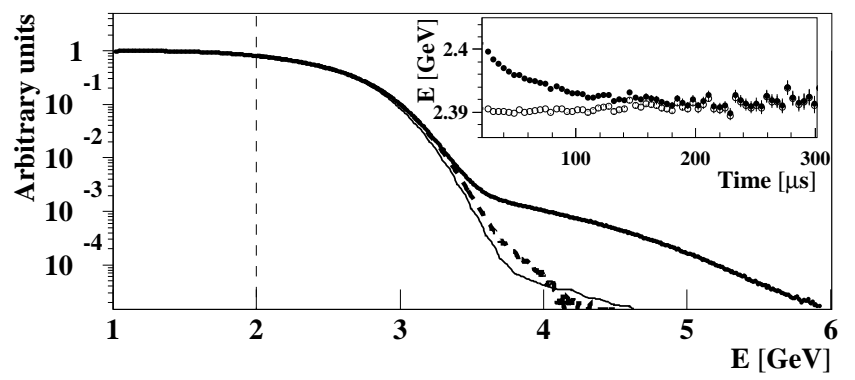

FIG. 2. The energy spectrum of the detected positrons above $1 \mathrm{GeV}$ at all times (thick line) and at only late times (thin line) together with the pileup-subtracted spectrum at all times (dashed line). The inset shows the energy above $2 \mathrm{GeV}$ averaged over one $g-2$ period as a function of time before (filled circles) and after (open circles) pileup subtraction for a typical detector.

(2) The storage ring is a weak focusing spectrometer (field index $n=0.137$ ) with an aperture which is large compared to the $18(\mathrm{w}) \times 57(\mathrm{~h}) \mathrm{mm}^{2}$ inflector aperture. Therefore, the phase space for the betatron oscillations defined by the acceptance of the storage ring is not filled. In combination with imperfect injection angles and an imperfect horizontal injection kick, this results in betatron oscillations of the beam as a whole - coherent betatron oscillations (CBO). These oscillations are observed directly using the fiber monitors. They also modulate the positron time spectra, since the acceptance of a calorimeter depends on the muon decay positions. The dominant effect is due to the horizontal oscillations, which decay with a time constant of $\sim 100 \mu$ s.

(3) The cyclotron period of the bunched beam leads to a strong modulation of the decay positron time spectra which remains at $32 \mu \mathrm{s}$ when we begin our fits. The modulation structure is eliminated from the time spectra by uniformly randomizing the start time for each detector and each storage fill over the range of one cyclotron period.

(4) Losses of muons during the data collection are minimized by controlled scraping [1] before the data collection is started. The magnitude and time-dependence of remaining losses are studied using coincident signals in the front scintillation counters of three adjacent calorimeters.

(5) Detector gain changes and time shifts are monitored with a pulsed laser system and also using the $e^{+}$ energy spectra. From $32 \mu \mathrm{s}$ on, the gains of the detectors except two are stable to within $0.1 \%$ over 10 dilated muon lifetimes. The reconstructed times are stable to within 20 ps over $200 \mu \mathrm{s}(0.1 \mathrm{ppm})$.

The raw WFD data were converted into $e^{+}$energies and times using two independent implementations of the pulse reconstruction algorithm. Four independent analyses of $\omega_{a}$ were made. For simplicity of presentation one will be described and the principal differences of the others will be discussed.

The pileup corrected time spectrum for the sum of the detectors is fitted by the function,

$$
f(t)=N(t) \cdot b(t) \cdot l(t) .
$$

Here, $N(t)$ is the muon decay and precession function of Eq. 2 and $b(t)$ is the coherent betatron oscillation function,

$$
b(t)=1+A_{b} e^{-t^{2} / \tau_{b}^{2}} \cos \left(\omega_{b} t+\phi_{b}\right),
$$

and $l(t)$ is the muon loss term,

$$
l(t)=1+n_{l} e^{-t / \tau_{l}} .
$$

The quantities $A_{b}, \tau_{b}, \omega_{b}$, and $\phi_{b}$ denote the CBO amplitude, time dependence, angular frequency, and phase, respectively, and $n_{l}$ and $\tau_{l}$ denote the fraction of lost muons and its time dependence. The CBO frequency $\omega_{b}$ is determined from a Fourier analysis of residuals in a fit of Eq. 2 to the data. The remaining 10 parameters in Eq. 3 are adjusted, in the sense of minimizing $\chi^{2}$. The frequency $\omega_{a}$ correlates strongly only to $\phi_{a}$. Consequently, $\omega_{a}$ is insensitive, unlike $\chi^{2}$, to the values of the other 8 parameters and to the functional forms of $b(t)$ and $l(t)$. Fig. 3 demonstrates the good agreement of data and fit, as indicated by $\chi^{2}=3818$ for 3799 degrees of freedom (dof).

The internal consistency of the results was verified in several ways. As an example, Fig. Ala shows the results when the fit range starts at increasing times after injection and $\omega_{a}$ is seen to be constant within statistical errors. Fig. $4 \mathrm{~b}$ shows the results for fits to the spectra from individual detectors $\left(\chi^{2} / \mathrm{dof}=30 / 21\right)$. The result for $\omega_{a}$ obtained from the average of individual detector fits (Fig. Wb) is consistent with the fit to the sum (Fig. (1a) to within $0.07 \mathrm{ppm}$. The fitted lifetime, after correcting for muon losses, agrees with that expected from special relativity to better than $0.1 \%$. 


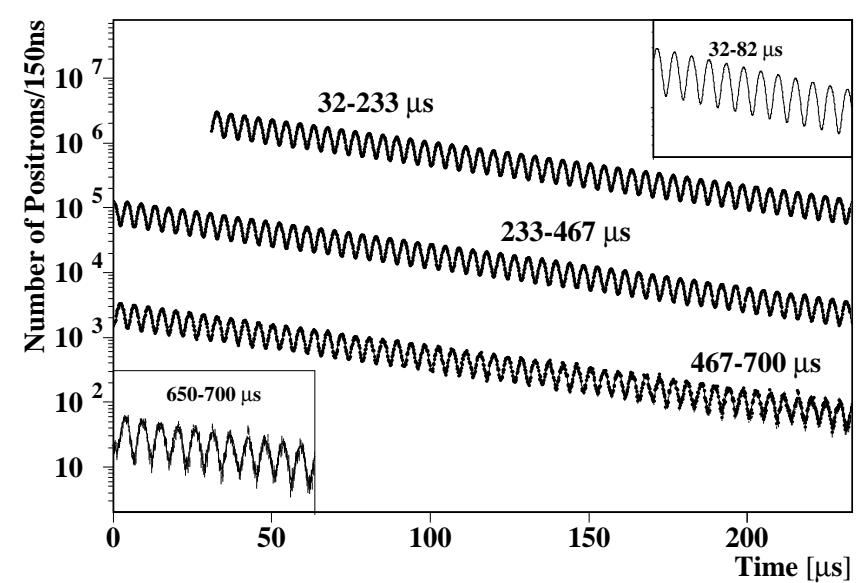

FIG. 3. Positron time spectrum overlaid with the fitted 10 parameter function $\left(\chi^{2} / \mathrm{dof}=3818 / 3799\right)$. The total event sample of $0.95 \times 10^{9} e^{+}$with $E \geq 2.0 \mathrm{GeV}$ is shown.
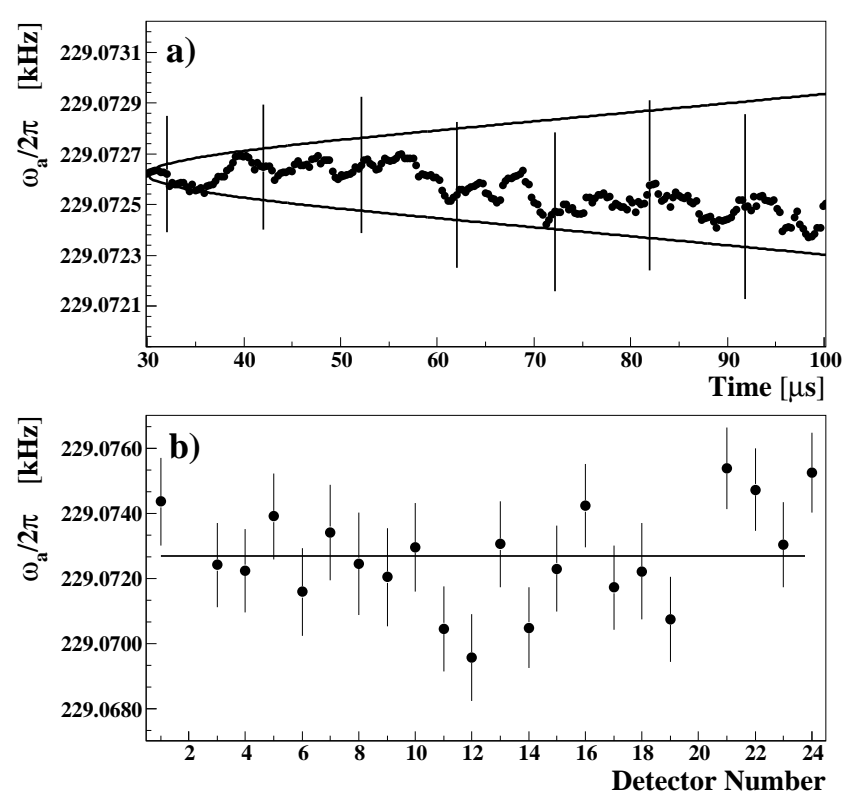

FIG. 4. a) The fitted frequency $\omega_{a} / 2 \pi$ vs. start time of fit is shown together with representative error bars. The band indicates the size of expected statistical fluctuations. b) Fits to the spectra from individual detectors. Detectors 2 and 20 were excluded from the $\omega_{a}$ analyses because of a readout problem and a miscalibration, respectively.

Two of the three other analyses used fitting functions similar to Eq. 3. The principal differences between the analysis described and the others consist of a somewhat different choice of data selection and fitting parameters, a refined treatment of detector gain changes, and alternative ways to determine pileup. In one analysis, a pileup correction was made by varying the minimum pulse separation time in the reconstruction algorithm, whereas in the other analysis pileup is incorporated in the fitted function. The pileup phase was fixed to the value obtained from the constructed pileup spectrum. In the fourth analysis, the data are randomly split in four samples $n_{1}-n_{4}$ which are rejoined in $u(t)=n_{1}(t)+n_{2}(t)$, $v(t)=n_{3}\left(t-\tau_{a} / 2\right)+n_{4}\left(t+\tau_{a} / 2\right)$, and the ratio

$$
r(t)=\frac{u(t)-v(t)}{u(t)+v(t)}=A(E) \sin \left(\omega_{a} t+\phi_{a}(E)\right)+\epsilon
$$

where $\tau_{a}$ is an estimate of the $g-2$ period and the constant $\epsilon \ll 1$. This ratio is largely independent of changes of observed counts on time scales larger than $\tau_{a}$, e.g. the muon lifetime. The ratio can thus be fitted with fewer free parameters and its results have somewhat different systematic uncertainties.

The results from the analyses are found to agree, on $\omega_{a}$ to within $0.3 \mathrm{ppm}$. This is within the statistical variation of $0.4 \mathrm{ppm}$ expected from the use of slightly different data in the respective analyses. We combined the results to $\omega_{a} / 2 \pi=229072.8 \pm 0.3 \mathrm{~Hz}(1.3 \mathrm{ppm})$. The only correction applied to our result was $+0.81 \pm 0.08 \mathrm{ppm}$ for the effects of the electric field and vertical betatron oscillations [1]. The error reflects the total uncertainty, accounts for the strong correlations between the individual results, and is dominated by the statistical contribution. The systematic errors are listed in Table II. The uncorrelated errors were added in quadrature, while the correlated errors were added linearly. Most of the systematics were common to all four analyses. Spin resonances, fit start time, and clock synchronization were considered and each was estimated to be less than $0.01 \mathrm{ppm}$.

TABLE II. Systematic errors for the $\omega_{a}$ analysis.

\begin{tabular}{l|c}
\hline \hline Source of errors & Size [ppm] \\
\hline Pileup & 0.13 \\
AGS background & 0.10 \\
Lost muons & 0.10 \\
Timing shifts & 0.10 \\
E field and vertical betatron oscillation & 0.08 \\
Binning and fitting procedure & 0.07 \\
Coherent betatron oscillation & 0.05 \\
Beam debunching/randomization & 0.04 \\
Gain changes & 0.02 \\
\hline Total systematic error on $\omega_{a}$ & 0.3 \\
\hline \hline
\end{tabular}

After the $\widetilde{\omega}_{p}$ and $\omega_{a}$ analyses were finalized, separately and independently, only then was the anomalous magnetic moment evaluated. The result is

$$
a_{\mu^{+}}=\frac{R}{\lambda-R}=11659202(14)(6) \times 10^{-10}(1.3 \mathrm{ppm})
$$

in which $R=\omega_{a} / \widetilde{\omega}_{p}, \mu_{\mu}=e \hbar\left(1+a_{\mu}\right) /\left(2 m_{\mu} c\right)$, and $\lambda=\mu_{\mu} / \mu_{p}=3.18334539(10)$ [8]. This new result is in good agreement with previous measurements [1.2,9] and reduces the combined error by a factor of about three.

The theoretical value of $a_{\mu}$ in the standard model (SM) [10] has its dominant contribution from quantum elec- 
trodynamics but the weak and strong interactions contribute as well. The value

$$
a_{\mu}(\mathrm{SM})=11659159.6(6.7) \times 10^{-10}(0.57 \mathrm{ppm})
$$

is the sum of $a_{\mu}(\mathrm{QED})=11658470.56(0.29) \times 10^{-10}$ $(0.025 \mathrm{ppm}), a_{\mu}($ weak $)=15.1(0.4) \times 10^{-10}(0.03 \mathrm{ppm})$, and $a_{\mu}(\mathrm{had})=673.9(6.7) \times 10^{-10}(0.57 \mathrm{ppm})$. The term $a_{\mu}(\mathrm{QED})$ is obtained using the value of $\alpha$ from $a_{e}(\exp )=a_{e}(\mathrm{SM})$ 10, and terms through order $\alpha^{5}$ are included. The term $a_{\mu}$ (weak) includes electroweak contributions of up to two-loop order. The term $a_{\mu}$ (had) arises from virtual hadronic contributions to the photon propagator in $4^{\text {th }}$ order and $6^{\text {th }}$ order, where the latter includes light-by-light scattering. The $4^{\text {th }}$ order term, which provides the largest contribution and uncertainty to $a_{\mu}$ (had) is obtained from measured hadron production cross sections in $e^{+} e^{-}$collisions and hadronic $\tau$ decay [11. Additional data on $e^{+} e^{-}$collisions from Novosibirsk 12 and from Beijing [13] and on $\tau$ decay from Cornell [14] have not yet been included in the evaluation of $a_{\mu}(\mathrm{had})$.

In Fig. : 5, the five most recent measurements of $a_{\mu}$ are shown along with the standard model prediction. The difference between the weighted mean of the experimental results, $a_{\mu}(\exp )=11659203(15) \times 10^{-10}(1.3 \mathrm{ppm})$, and the theoretical value from the standard model is

$$
a_{\mu}(\exp )-a_{\mu}(\mathrm{SM})=43(16) \times 10^{-10} .
$$

The error is the addition in quadrature of experimental and theoretical uncertainties. The difference is 2.6 times the stated error.

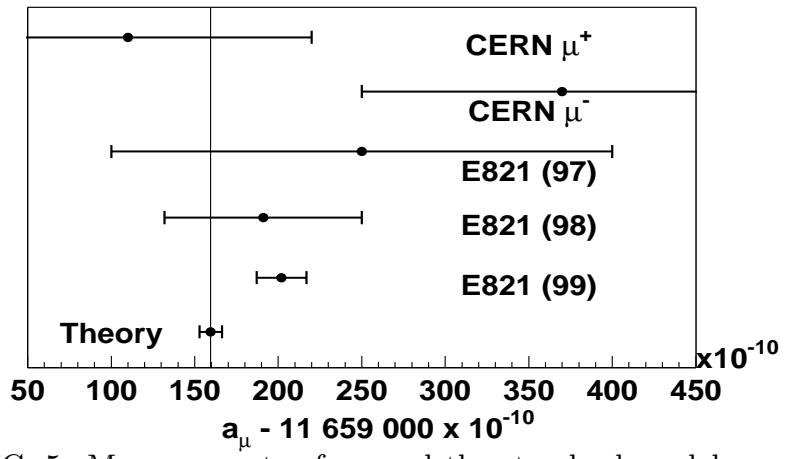

FIG. 5. Measurements of $a_{\mu}$ and the standard model prediction with their total errors.

Many speculative theories predict deviations from the standard model value for $a_{\mu}$ [10]. These include supersymmetry, muon substructure, and anomalous $W$ couplings. The muon anomalous $g$ value is particularly sensitive to supersymmetry [15] whose contributions to $a_{\mu}$ come from smuon-neutralino and sneutrino-chargino loops. In the limit of large $\tan \beta$, which is the ratio of the vacuum expectation values of two Higgs doublets, and for a degenerate spectrum of superparticles with mass $\widetilde{m}$,

$$
a_{\mu}(\mathrm{SUSY}) \approx 140 \times 10^{-11}\left(\frac{100 \mathrm{GeV}}{\widetilde{\mathrm{m}}}\right)^{2} \tan \beta .
$$

If we ascribe the difference $a_{\mu}(\exp )-a_{\mu}(\mathrm{SM})$ to $a_{\mu}$ (SUSY), for $\tan \beta$ in the range $4-40$, then $\widetilde{m} \approx 120$ $-400 \mathrm{GeV}$.

In 2000, approximately four times the total number of positrons were recorded as compared to our 1999 data. Measurements are now underway with negative muons, which will provide a sensitive test of CPT violation.

We thank T. Kirk, D.I. Lowenstein, P. Pile, and the staff of the BNL AGS for the strong support they have given this experiment. We thank D. Cassel, A. Czarnecki, M. Davier, T. Kinoshita, W. Marciano, and J. Urheim for helpful discussions. This work was supported in part by the U.S. Department of Energy, the U.S. National Science Foundation, the German Bundesminister für Bildung und Forschung, the Russian Ministry of Science, and the US-Japan Agreement in High Energy Physics.

[1] H.N. Brown et al., Muon $(g-2)$ Collaboration, Phys. Rev. D62, 091101 (2000).

[2] R.M. Carey et al., Muon $(g-2)$ Collaboration, Phys. Rev. Lett. 82, 1632 (1999).

[3] F. Krienen, D. Loomba and W. Meng, Nucl. Instrum. Methods A283, 5 (1989). A. Yamamoto et al., Proc. of 15th Int. Conf. on Magnet Technology, Science Press Beijing, 246 (1998).

[4] G.T. Danby, et al., Nucl. Instrum. Methods A457, 151 (2001).

[5] R. Prigl et al., Nucl. Instrum. Methods A374, 118 (1996)

[6] X. Fei et al. Nucl. Instrum. Methods A394, 349 (1997).

[7] S. Sedykh et al., Nucl. Instrum. Methods A455, 346 (2000).

[8] D.E. Groom et al., Review of Particle Physics, Eur. Phys. J. C15, 1 (2000).

[9] J. Bailey et al., Nucl. Phys. B150, 1 (1979).

[10] A. Czarnecki and W.J. Marciano, Nucl. Phys. (Proc. Suppl.) B76, 245 (1999).

[11] M. Davier and A. Höcker, Phys. Lett. B435, 427 (1998).

[12] R.R. Akhmetshin et al., CMD2 Collaboration, Phys. Lett. B475, 190 (2000).

[13] Z.G. Zhao, Int. J. Mod. Phys. A15, 3739 (2000) and J.Z. Bai, et al., BES Collaboration, Phys. Rev. Lett. 84, 594 (2000).

[14] S. Anderson et al., CLEO Collaboration Phys. Rev. D61, 112002 (2000).

[15] T. Moroi, Phys. Rev. D53, 6565 (1996). U. Chattopadhyay and P. Nath, Phys. Rev. D53, 1648 (1996). J.L. Lopez, D.V. Nanopoulos and X. Wang, Phys. Rev D49, 366 (1994). 